\title{
Super-Toda Theories and W-Algebras from Superspace Wess-Zumino-Witten Models
}

\author{
F. Delduc ${ }^{1}$, E. Ragoucy ${ }^{2}$, and P. Sorba ${ }^{1,2}$ \\ ${ }^{1}$ Laboratoire de Physique Theorique, ENS Lyon, 46 Allée d'Italie, F-69364 Lyon Cedex 07, \\ France \\ 2 Laboratoire de Physique Théorique, LAPP, Chemin de Bellevue, BP 110, \\ F-74941 Annecy-le-Vieux Cedex, France
}

Received October 20, 1991

\begin{abstract}
Using a superspace approach, it is proved that a $N=1$ Super-Toda theory can be seen as a constrained WZW model based on a supergroup. The gauge transformations which survive the constraints are then used $a$ la Drinfeld Sokolov to determine explicitly the super- $W$ algebra underlying this theory. The conformal spin content of any such super- $W$ algebra is provided in the general case.
\end{abstract}

\section{Introduction}

Rather recently, it has been remarked and exploited that Toda theories can be identified with some gauged Wess-Zumino-Witten (WZW) theories [1]. More precisely, a Toda theory can be seen as a WZW theory with constrained KacMoody currents, these constraints generating gauge transformations of special interest. Such a property is interesting for at least two reasons. First, because it relates two cornerstones of two dimensional conformal field theories, namely WZW models and Toda theories. Secondly, since this connection provides a direct method for the construction of $W$-algebra in Toda theories, the $W$-generators showing up as gauge invariant polynomials in the constrained currents and their derivatives.

It is the generalization of such properties to the $N=1$ supersymmetric case, namely the possible relation between supersymmetric Toda theories and superWZW models that we propose to examine in this paper. As is well known, a superToda theory is based on a simple superalgebra admitting a simple root system (S.R.S.) made only of fermionic roots [2]. Properties of super-Toda theories and some consequences for super- $W$ algebras have already been considered [3-5]. A first approach to recognize super-Toda theories in constrained super-WZW models based on supergroups has also been proposed in [6]. However, in this paper the WZW action is not explicitly supersymmetric, and the treatment, not

* URA 14-36 du CNRS, associée à l'E.N.S. de Lyon, et au L.A.P.P. 
being based on superfields but rather in their components, does not seem to be well adapted for the construction of super- $W$ algebras.

Hereafter, we will start from a manifestly supersymmetric WZW action associated with a supergroup $\mathscr{G}$. Mimicking the non-supersymmetric case, and imposing constraints on the supercurrent components associated with fermionic simple roots, we will obtain the $N=1$ super Toda theories. In order to make complete this gauge theory approach, we consider in a special paragraph the "component action," and recover the action given in [6] as the one associated to a Wess-Zumino gauge, obviously not covariant under supersymmetry (Sect. 2).

The general construction of $W$ components is considered in Sect. 3. Once again, we will develop a method analogous to the one used in [1] for the nonsupersymmetric case. The new feature here is that a super Toda theory based on a Lie superalgebra $\mathscr{A}$ involves the superprincipal embedding of $\operatorname{OSp}(1 \mid 2)$-we denote it $\mathrm{OSp}(1 \mid 2)_{\text {ppal }}$ - instead of the principal embedding of $\mathrm{Sl}(2)$ as it appears in a Toda theory based on an algebra. General formulas for the decomposition of any simple Lie superalgebra $\mathscr{A}$ with a completely fermionic S.R.S. into representations of its OSp $(1 \mid 2)_{\text {ppal }}$ are established in Sect. 4. From such tables, one can deduce the spin content of the $W$-generators associated with a $\mathscr{A}$-super-Toda theory (Sect. 5). Then, in Sect. 6, we illustrate our results on a few simple cases, before discussing further developments in the conclusion.

\section{W.Z.W. Action with a Supergroup, and Gauge Constraints}

We start this section by fixing some notations. Then, we write explicitly the W.Z.W. Lagrangian based on a supergroup in a manifestly supersymmetric framework. The adjunction of a second term to this expression will lead to the complete gauge invariant supersymmetric action associated with $N=1$ superToda theories. Finally, examining in some detail the components of the involved superfields, we can prove that the treatment presented in [6] and denoted "hidden supersymmetry" is obtained from an action through the use of a Wess-Zumino gauge.

\subsection{Some General Notations and Properties}

Super-Toda theories imply the use of special simple Lie superalgebras namely superalgebras $\mathscr{A}$ equipped with a completely fermionic simple root system (SRS) and admitting an OSp(1/2) principal embedding (see Sect. 3.2). They are of the following type $[2,7]$ :

$$
\begin{gathered}
\operatorname{Sl}(n+1 \mid n), \operatorname{OSp}(2 n \pm 1 \mid 2 n), \operatorname{OSp}(2 n \mid 2 n), \\
\operatorname{OSp}(2 n+2 \mid 2 n) n \geqq 1 \text { and } D(2,1 ; \alpha) \alpha \neq 0,-1 .
\end{gathered}
$$

For brevity, we will call these superalgebras "fermionic" superalgebras".

By $\langle$,$\rangle we denote in \mathscr{A}$ the unique (up to a constant factor) non-degenerate, invariant, supersymmetric bilinear form; such a form exists for any contragredient - or basic - simple Lie superalgebra [7]. In the Chevalley basis, the Cartan generators $H_{i}(i=1, \ldots, r=\operatorname{rank}(A))$ and the fermionic generators $E_{ \pm \alpha_{i}}$ associated to

1 Note that although each superalgebra $\operatorname{PSl}(n \mid n) \equiv \operatorname{Sl}(n \mid n) / U(1), n \neq 1$, possesses a fermionic SRS, it does not give rise to an OSp(1/2) principal embedding 
the simple root system $\Delta_{+}=\left\{\alpha_{1}, \ldots, \alpha_{r}\right\}$ satisfy the Commutation Relations (C.R.):

$$
\begin{aligned}
& {\left[H_{i}, E_{ \pm \alpha_{j}}\right]= \pm K_{i j} E_{ \pm \alpha_{j}},} \\
& \left\{E_{+\alpha_{i}}, E_{-\alpha_{j}}\right\}=\delta_{i j} H_{i}, \\
& {\left[H_{i}, H_{j}\right]=0,}
\end{aligned}
$$

where $K$ is the (symmetric [8]) Cartan matrix defined by

$$
K_{i j}=\left(\alpha_{i}, \alpha_{j}\right) \equiv\left\langle H_{i}, H_{j}\right\rangle \text {. }
$$

Moreover one has

$$
\begin{aligned}
& \left\langle H_{i}, E_{ \pm \alpha_{j}}\right\rangle=\left\langle E_{ \pm \alpha_{i}}, E_{ \pm \alpha_{j}}\right\rangle=0, \\
& \left\langle E_{\alpha_{i}}, E_{-\alpha_{j}}\right\rangle=\delta_{i j} .
\end{aligned}
$$

We will denote by $K^{i j}$ the entries of the inverse Cartan matrix, i.e.:

$$
K^{i j} K_{j k}=\delta_{k}^{i} \text {. }
$$

General results on superalgebras and their Dynkin diagrams can also be found in [9].

\subsection{Gauged Wess-Zumino-Witten Action}

The light-cone coordinates of the world-sheet $(1,1)$ superspace will be denoted by $x^{++}, x^{--}, \eta^{+}, \eta^{-}$. The index refers to the Lorentz weight carried by the coordinate. The supersymmetric covariant derivatives $D_{ \pm}$satisfy

$$
\left(D_{ \pm}\right)^{2}=i \partial_{ \pm \pm},\left\{D_{+}, D_{-}\right\}=0 \text {. }
$$

The superfield $G(x, \eta)$ belongs to a supergroup $\mathscr{G}$ such that the corresponding superalgebra $\mathscr{A}$ admits a set of simple roots all fermionic. We shall denote by ${ }^{\wedge}$ the automorphism of the superalgebra $\mathscr{A}$ which changes the sign of anticommuting generators and leaves commuting generators unchanged. The same notation will be used for the corresponding automorphism of the supergroup. The starting point is the WZW action

$$
\begin{aligned}
S_{0}(G)= & \frac{\kappa}{2} \int d^{2} x d^{2} \eta\left[\left\langle\left(G^{-1} D_{+} \hat{G}\right),\left(\hat{G}^{-1} D_{-} G\right)\right\rangle\right. \\
& \left.+\int d t\left\langle\left(G^{-1} \partial_{t} G\right),\left(\left(G^{-1} D_{+} \hat{G}\right)\left(\hat{G}^{-1} D_{-} G\right)+\left(G^{-1} D_{-} \hat{G}\right)\left(\hat{G}^{-1} D_{+} G\right)\right)\right\rangle\right] .
\end{aligned}
$$

The corresponding equations of motion read

$$
D_{-}\left(\hat{G}^{-1} D_{+} G\right)=0 \Leftrightarrow D_{+}\left(D_{-} G G^{-1}\right)=0 .
$$

The action (2.5) satisfies the Polyakov-Wiegmann relation

$$
S_{0}\left(G_{1} G_{2}\right)=S_{0}\left(G_{1}\right)+S_{0}\left(G_{2}\right)+\kappa \int d^{2} x d^{2} \eta\left\langle\left(G_{1}^{-1} D_{+} \hat{G}_{1}\right),\left(D_{-} G_{2} G_{2}^{-1}\right)\right\rangle .
$$

We denote by $\mathscr{A}_{+}$(respectively $\mathscr{A}_{-}$) the superalgebras spanned by the generators of $\mathscr{A}$ corresponding to positive (respectively negative) roots with respect to the Cartan subalgebra $\mathscr{H}$. The supergroups corresponding to $\mathscr{A}_{+}\left(\mathscr{A}_{-}\right)$will be denoted by $\mathscr{G}_{+}\left(\mathscr{G}_{-}\right)$. In order to gauge the left action of $\mathscr{G}_{+}$and the right action of 
$\mathscr{G}_{-}$, we make the transformation

$$
G \rightarrow \alpha G \beta,
$$

where $\alpha$ and $\beta$ are superfields belonging respectively to $\mathscr{G}_{+}$and $\mathscr{G}_{-}$. Then one finds:

$$
\begin{aligned}
& S_{0}(\alpha G \beta)=S_{0}(G)+\kappa \int d^{2} x d^{2} \eta\left[\left\langle\left(\alpha^{-1} D_{+} \hat{\alpha}\right),\left(D_{-} G G^{-1}\right)\right\rangle\right. \\
& \left.\quad+\left\langle\left(G^{-1} D_{+} \hat{G}\right),\left(D_{-} \beta \beta^{-1}\right)\right\rangle+\left\langle G^{-1}\left(\alpha^{-1} D_{+} \hat{\alpha}\right) \hat{G},\left(D_{-} \beta \beta^{-1}\right)\right\rangle\right] .
\end{aligned}
$$

One can introduce the gauge superfields

$$
A_{+}=\alpha^{-1} D_{+} \hat{\alpha} \in \mathscr{A}_{+}, A_{-}=D_{-} \beta \beta^{-1} \in \mathscr{A}_{-} .
$$

Moreover, we introduce the constant elements $\mu \in \mathscr{A}_{-}$and $v \in \mathscr{A}_{+}$which have non-zero elements along (fermionic) simple roots only:

$$
\mu=\sum_{i=1}^{r} \mu_{i} E_{-\alpha_{i}}, v=\sum_{i=1}^{r} v_{i} E_{\alpha_{i}} .
$$

Then the complete gauge-invariant superspace action which leads to the supersymmetric Toda models is

$$
\begin{aligned}
S\left(G, A_{+}, A_{-}\right)= & S_{0}(G)+\kappa \int d^{2} x d^{2} \eta\left[\left\langle A_{+},\left(D_{-} G G^{-1}-\mu\right)\right\rangle\right. \\
& \left.+\left\langle\left(G^{-1} D_{+} \hat{G}-v\right), A_{-}\right\rangle+\left\langle G^{-1} A_{+} \hat{G}, A_{-}\right\rangle\right],
\end{aligned}
$$

and the infinitesimal gauge transformations of the superfields are

$$
\begin{aligned}
& \delta_{\Lambda, \Omega} G=\Lambda G+G \Omega, \\
& \delta_{\Lambda, \Omega} \alpha=-\alpha \Lambda \Rightarrow \delta_{\Lambda, \Omega} A_{+}=-D_{+} \hat{\Lambda}+\Lambda A_{+}-A_{+} \hat{\Lambda}, \\
& \delta_{\Lambda, \Omega} \beta=-\Omega \beta \Rightarrow \delta_{\Lambda, \Omega} A_{-}=-D_{-} \Omega+A_{-} \Omega-\hat{\Omega} A_{-},
\end{aligned}
$$

where the superfield $\Lambda$ belongs to the algebra $\mathscr{A}_{+}$and the superfield $\Omega$ belongs to $\mathscr{A}_{-}$.

\subsection{Component Action}

When turning to components, we shall use the superspace gauge freedom to eliminate all components of the superfields which transform into a supergauge parameter without space-time derivatives. Thus, from the transformation laws

$$
\left.\delta_{\Lambda, \Omega} A_{+}\right|_{\eta=0}=\left.D_{+} \hat{\Lambda}\right|_{\eta=0}+\ldots,\left.\delta_{\Lambda, \Omega}\left(D_{-} A_{+}\right)\right|_{\eta=0}=\left.\left(D_{-} D_{+} \hat{\Lambda}\right)\right|_{\eta=0}+\ldots,
$$

we deduce that one can find a gauge such that

$$
\left.A_{+}\right|_{\eta=0}=0,\left.\left(D_{-} A_{+}\right)\right|_{\eta=0}=0 \text {. }
$$

In the same way, one can choose

$$
\left.A_{-}\right|_{\eta=0}=0,\left.\left(D_{+} A_{-}\right)\right|_{\eta=0}=0 .
$$

We are then left with the physical gauge fields defined by

$$
A_{++}(x)=\left.\left(D_{+} \hat{A}_{+}\right)\right|_{\eta=0}, A_{--}(x)=\left.\left(D_{-} A_{-}\right)\right|_{\eta=0},
$$

and with the auxiliary fields

$$
\xi_{+}=\left.\left(D_{-} D_{+} A_{+}\right)\right|_{\eta=0}, \xi_{-}=\left.\left(D_{-} D_{+} A_{-}\right)\right|_{\eta=0} .
$$


Then we define the components of the superfield $G(x, \eta)$ by

$$
g(x)=\left.G\right|_{\eta=0}, \psi_{-}=\left.\left(D_{-} G G^{-1}\right)\right|_{\eta=0}, \psi_{+}=\left.\left(G^{-1} D_{+} \hat{G}\right)\right|_{\eta=0} .
$$

The auxiliary fields at the second level in $\eta$ play no role in the sequel and will be ignored. The gauge transformation laws of $\psi_{-}$and $\psi_{+}$are as follows:

$$
\delta_{\Lambda, \Omega} \psi_{-}=\left.\left(D_{-} \Lambda\right)\right|_{\eta=0}+\ldots, \delta_{\Lambda, \Omega} \psi_{+}=\left.\left(D_{+} \hat{\Omega}\right)\right|_{\eta=0}+\ldots
$$

These components of the superfield parameters have not been used in $(2.12,2.13)$, and one can thus choose a gauge such that

$$
\left.\psi_{-}\right|_{\mathscr{A}_{+}}=0,\left.\psi_{+}\right|_{\mathscr{A}_{-}}=0 \text {. }
$$

We have now used all the parameters of the supergauge transformations beside the ordinary gauge parameters $\lambda(x)=\left.\Lambda\right|_{\eta=0}$ and $\omega(x)=\left.\Omega\right|_{\eta=0}$. Finally, it is easy to see that the auxiliary fields $\xi_{+}$and $\xi_{-}$enter into the action through

$$
-\kappa \int d^{2} x d^{2} \eta\left[\left\langle\xi_{+},\left(\psi_{-}-\mu\right)\right\rangle+\left\langle\left(\psi_{+}-v\right), \xi_{-}\right\rangle\right] .
$$

Remembering that $\xi_{+}$belongs to $\mathscr{A}_{+}$and $\xi_{-}$to $\mathscr{A}_{-}$, we see that these fields are Lagrange multipliers for the constraints

$$
\psi_{-\mathscr{A}_{-}}=\mu,\left.\psi_{+}\right|_{\mathscr{A}_{+}}=v .
$$

As a result of $(2.14,2.15), \psi_{-}$and $\psi_{+}$reduce to

$$
\psi_{-}=\chi_{-}+\mu, \psi_{+}=\chi_{+}+v,
$$

where $\chi_{+}$and $\chi_{-}$belong to the Cartan subalgebra $\mathscr{H}$. Then the component form of the action is

$$
\begin{aligned}
S= & \frac{\kappa}{2} \int d^{2} x\left\langle g^{-1} \partial_{++} g, g^{-1} \partial_{--} g\right\rangle+\int d t\left\langle g^{-1} \partial_{t} g,\left[g^{-1} \partial_{++} g, g^{-1} \partial_{--} g\right]\right\rangle \\
& +i\left\langle\chi_{-}, \partial_{++} \chi_{-}\right\rangle+i\left\langle\chi_{+}, \partial_{-} \chi_{+}\right\rangle-2\left\langle A_{++},\left(i \partial_{--} g g^{-1}-\mu^{2}+\left[\chi_{-}, \mu\right]\right)\right\rangle \\
& \left.-2\left\langle\left(i g^{-1} \partial_{++} g+v^{2}-\left[\chi_{+}, v\right]\right), A_{--}\right\rangle-2\left\langle g^{-1} A_{++} g, A_{--}\right\rangle\right]
\end{aligned}
$$

with the following gauge transformation laws:

$$
\begin{aligned}
& \delta_{\lambda, \omega} g=\lambda g+g \omega, \delta_{\lambda, \omega} \chi_{-}=-\{\hat{\lambda}, \mu\}, \delta_{\lambda, \omega} \chi_{+}=-\{\hat{\omega}, v\}, \\
& \delta_{\lambda, \omega} A_{++}=-i \partial_{++} \hat{\lambda}-\left[A_{++}, \hat{\lambda}\right], \delta_{\lambda, \omega} A_{--}=-i \partial_{--} \omega+\left[A_{--}, \omega\right],
\end{aligned}
$$

where $\hat{\lambda}$ (respectively $\hat{\omega}$ ) is the restriction of $\lambda$ (respectively $\omega$ ) to anticommuting generators corresponding to positive (respectively negative) simple roots. The action (2.16) coincides with the one given in [6]. In particular, the constraints derived from the $A_{++}$equations of motion are

$$
\left.i \partial_{--} g g^{-1}\right|_{\mathscr{A}_{-}}=\frac{1}{2}\{\mu, \mu\}-\left[\chi_{-}, \mu\right]-g A_{--} g^{-1} .
$$

In the gauge $A_{--}=0$, we recover the constraints of [6] including the fermions $\chi_{-}$, in which the non-zero currents correspond to the anticommutators of two simple roots.

Thus, the origin of the supersymmetry of their action is now clear: it is just an ordinary supersymmetric action in a gauge which is not covariant with respect to supersymmetry. The supersymmetry transformations of the fields are easily 
obtained from the transformations of the superfields. One finds

$$
\begin{aligned}
& \delta g=\varepsilon^{-} \psi_{-} g+\varepsilon^{+} \hat{g} \hat{\psi}_{+}, \\
& \delta \psi_{-}=\left.\varepsilon^{-}\left(i \partial_{--} g g^{-1}+g A_{--} g^{-1}\right)\right|_{\mathscr{H}}, \delta \psi_{+}=\left.\varepsilon^{+}\left(i g^{-1} \partial_{++} g+g^{-1} A_{++} g\right)\right|_{\mathscr{H}}, \\
& \delta A_{++}=\left.\varepsilon^{-}\left(\psi_{-} A_{++}-\hat{A}_{++} \psi_{-}\right)\right|_{\mathscr{A}+}, \delta A_{--}=-\left.\varepsilon^{+}\left(\hat{\psi}_{+} A_{--}-\hat{A}_{--} \hat{\psi}_{+}\right)\right|_{\mathscr{A}} .
\end{aligned}
$$

It takes some time to check that these transformations are an invariance of the action (2.16), and also that the commutator of two such transformations with parameters $\varepsilon$ and $\varepsilon^{\prime}$ closes, up to field equations, on a translation and on a field dependent gauge transformation

$$
\left[\delta, \delta^{\prime}\right] g=2 i\left(\varepsilon^{\prime-} \varepsilon^{-} \partial_{--} g+\varepsilon^{\prime+} \varepsilon^{+} \partial_{++} g\right)+\delta_{\lambda, \omega} g
$$

with

$$
\begin{aligned}
& \lambda=-\left.2 \varepsilon^{\prime-} \varepsilon^{-}\left(i \partial_{-} g g^{-1}+g A_{--} g^{-1}\right)\right|_{\mathscr{A}+}+2 \varepsilon^{\prime+} \varepsilon^{+} A_{++}, \\
& \omega=-\left.2 \varepsilon^{\prime+} \varepsilon^{+}\left(i g^{-1} \partial_{++} g+g^{-1} A_{++} g\right)\right|_{\mathscr{A}_{-}}+2{\varepsilon^{\prime}}^{-} \varepsilon^{-} A_{-} \text {. }
\end{aligned}
$$

\section{Super-Toda Theories and Super- $W$ Algebras}

Here we will use the gauge invariance properties of the action given in Eq. (2.10) to recognize the $N=1$ Super-Toda theories. Then the construction of the $W$ elements associated to such theories will be achieved by generalizing in the supersymmetric case the Drinfeld-Sokolov gauge used in [10].

\subsection{Constraints and Super-Toda Action}

Coming back to the gauged WZW action (2.10), one can write down the corresponding equations of motion. Then, making use of the gauge invariance and setting $A_{+}=A_{-}=0$, one gets as Euler equations those of the super-WZW model

$$
D_{-}(\bar{J})=0 \Leftrightarrow D_{+}(J)=0,
$$

where $\bar{J}$ and $J$ are the supercurrents

$$
J=\kappa D_{-} G G^{-1}, \bar{J}=\kappa \hat{G}^{-1} D_{+} G
$$

together with the constraints

$$
\begin{aligned}
& \left\langle E_{\alpha}, D_{-} G G^{-1}-\mu\right\rangle=0, \\
& \left\langle E_{-\alpha}, \hat{G}^{-1} D_{+} G+v\right\rangle=0
\end{aligned}
$$

for any $\alpha \in \Pi_{+}=$set of positive (bosonic and fermionic) roots. That is also, using relations (2.9):

$$
\begin{gathered}
\left\langle E_{\alpha_{i}}, D_{-} G G^{-1}\right\rangle=\mu_{i},\left\langle E_{\alpha}, D_{-} G G^{-1}\right\rangle=0, \\
\left\langle E_{-\alpha_{i}}, \hat{G}^{-1} D_{+} G\right\rangle=-v_{i},\left\langle E_{-\alpha}, \hat{G}^{-1} D_{+} G+v\right\rangle=0,
\end{gathered}
$$

with $\alpha_{i} \in \Delta_{+}$and $\alpha \in \Pi_{+} \backslash \Delta_{+}$. Recalling that $\mu_{i}$ and $v_{i}$ are constant quantities, these constraints are obviously the supersymmetric analogues of the ones of [1]. 
In order to recognize in the Lagrangian, as well as in the equations of motion, the $N=1$ super-Toda theory, let us restrict ourselves to supergroup valued superfields $G$ admitting a local Gauss decomposition

$$
G=A B C
$$

with

$$
A=\exp \left(\sum_{\alpha \in \boldsymbol{\Pi}_{+}} X^{\alpha} E_{\alpha}\right), B=\exp \left(\sum_{i=1}^{r} \Phi^{i} H_{i}\right), C=\exp \left(\sum_{\alpha \in \Pi_{+}} Y^{\alpha} E_{-\alpha}\right),
$$

where $E_{ \pm \alpha}$ is the bosonic or fermionic generator associated to the root $\pm \alpha \in \Pi_{+}$. The $\Phi^{i}\left(x^{++}, x^{--}, \eta^{+}, \eta^{-}\right)$- denoted for simplicity by $\Phi$-are bosonic superfields, while the $X^{\alpha}$ and $Y^{\alpha}$ are bosonic (respectively fermionic) local superfields following the corresponding generator $E_{ \pm \alpha}$ is commuting (respectively anticommuting).

From the constraints (3.4) and (3.5) we deduce the equations

$$
\begin{aligned}
& A^{-1} D_{+} A=\sum_{\alpha_{i} \in \Delta_{+}} v_{i} E_{\alpha_{i}} \exp \left(\sum_{\alpha_{j} \in \Delta_{+}} K_{i j} \Phi^{j}\right), \\
& D_{-} C C^{-1}=\sum_{\alpha_{i} \in \Delta_{+}} \mu_{i} E_{-\alpha_{i}} \exp \left(\sum_{\alpha_{j} \in \Delta_{+}} K_{i j} \Phi^{j}\right),
\end{aligned}
$$

which, together with the Polyakov-Wiegmann relation (2.7), lead to the action

$$
S(G)=\int d^{2} x d^{2} \eta\left\{-K_{i j} D_{-} \Phi^{i} D_{+} \Phi^{j}+\sum_{i=1}^{r}\left(\mu_{i} v_{i}\right) \exp \left(K_{i j} \Phi^{j}\right)\right\},
$$

where $r$ is the rank of the supergroup $\mathscr{G}$. The explicit form of the equations of motion can be calculated from (3.10): one gets the $r=\operatorname{rank}(\mathscr{G})$ equations of the $N=1 \mathscr{G}$-super Toda theory

$$
D_{-} D_{+} \Phi^{i}+\left(\mu_{i} v_{i}\right) \exp \left(K_{i j} \Phi^{j}\right)=0(i=1, \ldots, r) .
$$

Now it is a simple exercise to verify that the invariance group of the constraints (3.4) (respectively (3.5)) is exactly generated by the elements $\varrho\left(x^{--}, \eta^{-}\right) \in \mathscr{G}_{+}$ (respectively $\left.\sigma\left(x^{++}, \eta^{+}\right) \in \mathscr{G}_{-}\right)$. These residual gauge transformations act on the currents as

$$
\begin{gathered}
J \rightarrow \hat{\varrho} J \varrho^{-1}+\left(D_{-} \varrho\right) \varrho^{-1}=\operatorname{Ad}^{*}(\varrho)(J), \\
\bar{J} \rightarrow \hat{\sigma}^{-1} \bar{J} \sigma+\hat{\sigma}^{-1}\left(D_{+} \sigma\right)=\operatorname{Ad}^{*}\left(\sigma^{-1}\right)(\bar{J}) .
\end{gathered}
$$

\subsection{The Algebra $\mathrm{OSp}(1 \mid 2)$ and Super-Toda Theories}

It is known [11] that the superalgebra OSp(1|2) must play in Super-Toda theories a role analogous to the one played by Sl(2) in Toda theories [2]. This will be seen explicitly in the following.

Let us concentrate on the supercurrent $J=J\left(x^{--}, \eta^{-}\right)$. The constraints (3.4) impose $J$ to be of the form

$$
J\left(x^{--}, \eta^{-}\right)=J_{-\frac{1}{2}}+\sum_{\alpha_{i} \in \Delta_{+}} \Phi^{i}\left(x^{--}, \eta^{-}\right) H^{i}+\sum_{\alpha \in \Pi_{+}} X_{\alpha}\left(x^{--}, \eta^{-}\right) E_{\alpha},
$$

where we now call for convenience $J_{-\frac{1}{2}}$ the quantity $\mu$ defined in (2.9)

$$
J_{-\frac{1}{2}} \equiv \mu=\sum_{i=1}^{r} \mu_{i} E_{-\alpha_{i}} .
$$


Then, defining

$$
J_{+\frac{1}{2}}=\sum_{i=1}^{r} \frac{1}{\mu_{i}}\left(\sum_{j=1}^{r} K^{i j}\right) E_{\alpha_{i}}
$$

and

$$
H=\frac{1}{2}\left\{J_{-\frac{1}{2}}, J_{+\frac{1}{2}}\right\}=\frac{1}{2} \sum_{i=1}^{r} K^{i j} H_{j},
$$

it is straightforward to verify that

$$
\left[H, J_{ \pm \frac{1}{2}}\right]= \pm \frac{1}{2} J_{ \pm \frac{1}{2}} .
$$

Defining also

$$
X_{ \pm}=\frac{1}{2}\left\{J_{ \pm \frac{1}{2}}, J_{ \pm \frac{1}{2}}\right\}
$$

we can check that these last quantities are not zero since

$$
\left[J_{ \pm}, J_{\mp \frac{1}{2}}\right]=-2\left[H, J_{ \pm \frac{1}{2}}\right]=\mp J_{ \pm \frac{1}{2}}
$$

by using the Jacobi identity. Finally the C.R.

$$
\left[J_{\mp \frac{1}{2}}, X_{\mp}\right]=0,\left[H, X_{ \pm}\right]= \pm X_{ \pm}
$$

complete the proof that generators $\left\{J_{ \pm \frac{1}{2}}, X_{ \pm}, H\right\}$ form a basis of an $\operatorname{OSp}(1 \mid 2)$ algebra. We note that this superalgebra constructed from (3.15) and (3.16) as a subalgebra of the $\mathscr{A}$ superalgebra, appears as the superprincipal embedding of $\operatorname{OSp}(1 \mid 2)$ in $\mathscr{A}:$ we denote it $\mathrm{OSp}(1 \mid 2)_{\text {ppal }}$ (as already mentioned in [1] for the case of algebras, the values of the $\mu_{i}$ parameters can be chosen equal to unity by rescaling the step operators $E_{ \pm \alpha_{i}}$ ).

Let us emphasize that, following (3.18) and (3.21), $J_{ \pm \frac{1}{2}}$ and $X_{ \pm}$are eigenstates of $H$ with the respective eigenvalues $h= \pm \frac{1}{2}$ and $h= \pm 1$. Remembering the C.R. (2.2) any $E_{ \pm \alpha_{i}}$ with $\alpha_{i} \in \Delta_{+}$satisfies

$$
\left[H, E_{ \pm \alpha_{i}}\right]= \pm \frac{1}{2} \sum_{k=1}^{r} K^{k j} K_{j i} E_{ \pm \alpha_{i}}= \pm \frac{1}{2} E_{ \pm \alpha_{i}} .
$$

From the decomposition of any root from simple roots and the Jacobi identity, we deduce that $H$ provides a grading of the $\mathscr{A}$ superalgebra

$$
\left[H, E_{h}\right]=h E_{h} \text { with } h \in \frac{1}{2} \mathbf{Z} .
$$

Each fermionic root belongs to an $H$-eigenspace $\mathscr{A}^{(h)}$ with $h$ half-integer, while each bosonic root corresponds to an integer value of $h$. Denoting by $\phi=\sum_{i=1}^{r} a_{i} \alpha_{i}$ the highest root of $\mathscr{A}$, the highest value of $h$ in $\mathscr{A}$ will be

$$
h_{\max }=\frac{1}{2} \sum_{i=1}^{r} a_{i}
$$

and we have

$$
\begin{array}{cccc}
{\left[\mathscr{A}^{\left(h_{1}\right)}, \mathscr{A}^{\left(h_{2}\right)}\right] \subset \mathscr{A}^{\left(h_{1}+h_{2}\right)}} & \text { if } & \left|h_{1}+h_{2}\right| \leqq h_{\max } \\
=\emptyset & \text { if } & \left|h_{1}+h_{2}\right|>h_{\max }
\end{array}
$$




\subsection{Determination of the Super-W Generators}

We are now in position to generalize to superalgebras the Drinfeld-Sokolov techniques for the characterization of $W$-generators. The power of this approach for Lie algebras has been made explicit in [1].

Let us rewrite (3.14) as

$$
J=\sum_{h=-\frac{1}{2}}^{h_{\max }} J_{h},
$$

$J_{0}$ being associated with the Cartan part and $J_{h}(h>0)$ to the roots of $\Pi^{(h)}=\left\{\alpha \in \Pi_{+} / E_{\alpha} \in \mathscr{A}^{(h)}\right\}$. Now, consider a $\mathscr{G}$-element of the form:

$$
g_{h_{0}}=\exp \left(\sum_{\alpha \in \Pi^{\left(h_{0}\right)}} \Omega^{\alpha} E_{\alpha}\right) h_{0} \geqq \frac{1}{2} .
$$

One easily notes that

$$
\left(\operatorname{Ad}^{*}\left(g_{h_{0}}\right)(J)-J\right) \in \underset{h \geqq h_{0}-\frac{1}{2}}{\bigoplus} \mathscr{A}^{(h)}
$$

and the restriction of this quantity to $\mathscr{A}^{\left(h_{0}-\frac{1}{2}\right)}$ is a linear function of the parameters $\Omega^{\alpha}\left(x^{--}, \eta^{-}\right)$of $g_{h_{0}}$,

$$
\left.\left(\operatorname{Ad}^{*}\left(g_{h_{0}}\right)(J)-J\right)\right|_{\mathscr{A}\left(h_{0}-\frac{1}{2}\right)}=\sum_{\alpha \in \Pi^{\left(h_{0}\right)}} \Omega^{\alpha}\left[E_{\alpha}, J_{-\frac{1}{2}}\right] .
$$

Then it is natural to look for an element $g_{h_{0}}$ annihilating the largest number of terms in $J_{h_{0}-\frac{1}{2}}$. Repeating the process for the different values of $h_{0} \geqq \frac{1}{2}$, we will be left with elements belonging to the $\mathscr{A}$ subspaces

$$
\mathscr{N}^{\left(h_{0}\right)}=\mathscr{A}^{\left(h_{0}\right)} \backslash J_{-\frac{1}{2}}\left(\mathscr{A}^{\left(h_{0}+\frac{1}{2}\right)}\right)
$$

with

$$
J_{-\frac{1}{2}}\left(\mathscr{A}^{\left(h_{0}+\frac{1}{2}\right)}\right)=\left[J_{-\frac{1}{2}}, \mathscr{A}^{\left(h_{0}+\frac{1}{2}\right)}\right] .
$$

As it will be explicitly shown in the next section, if

$$
n_{h_{0}}=\operatorname{dim} \mathscr{N}^{\left(h_{0}\right)}=\operatorname{dim} \mathscr{A}^{\left(h_{0}\right)}-\operatorname{dim} J_{-\frac{1}{2}}\left(\mathscr{A}^{\left(h_{0}+\frac{1}{2}\right)}\right),
$$

one has

$$
\sum_{h \geqq \frac{1}{2}} n_{h}=r=\operatorname{rank}(\mathscr{A})
$$

thus a suitable group element

$$
g=g_{h_{\max }} g_{h_{\max }-\frac{1}{2}} \cdots g_{\frac{1}{2}}
$$

will allow to gauge transform $J$ into

$$
J^{g}=\operatorname{Ad}^{*}(g)(J)=J_{-\frac{1}{2}}+\sum_{i=1}^{r} W_{h_{i}} P^{\left(h_{i}\right)}
$$

with $P^{\left(h_{i}\right)} \in \mathscr{N}^{\left(h_{i}\right)}, \frac{1}{2} \leqq h_{i} \leqq h_{\max }$. Due to the complete reducibility of the $\mathscr{A}$ superalgebra into OSp $(1 \mid 2)_{\text {ppal }}$ supermultiplets (see Sect. 4), the $r$ elements $P^{\left(h_{i}\right)}$ we have to select in the $\mathscr{N}^{\left(h_{i}\right)}$ can be chosen such that

$$
J_{+\frac{1}{2}}\left(P^{\left(h_{i}\right)}\right)=0, h_{i} \geqq \frac{1}{2} ; i=1, \cdots, r .
$$


Following [1] we will call such particular Drinfeld-Sokolov gauge the highest weight gauge.

The $W_{h_{i}}$ quantities are differential polynomials in the components of the currents $J\left(x^{--}, \eta^{-}\right)$. Their construction ensures that they form a complete set of gauge invariant polynomials, so that they will close under the Poisson Brackets (PB) of the theory and constitute a super- $W$ algebra. To determine the spin content of this algebra (Sect. 5), we have first to reduce the adjoint representation of each fermionic superalgebra with respect to its $\operatorname{OSp}(1 \mid 2)_{\text {ppal }}$ (Sect.4).

\section{OSp(1|2) $)_{\text {ppal }}$ Multiplets in Fermionic Simple Superalgebras}

In this section we study the decomposition of a fermionic simple superalgebra $\mathscr{A}$ into irreducible representations of its $\operatorname{OSp}(1 \mid 2)_{\text {ppal }}$ subalgebra. Before presenting the results, let us recall some general properties of $\mathrm{OSp}(1 \mid 2)$ superalgebra.

\subsection{Some Properties of the $\mathrm{OSp}(1 \mid 2)$ Superalgebra}

The following features about $\operatorname{OSp}(1 \mid 2)$ will be useful for the rest of the section.

Proposition 1 [12]. All finite dimensional representations of $\mathrm{OSp}(1 \mid 2)$ are completely reducible.

This ensures that the adjoint representation of a superalgebra $\mathscr{A}$ reduces to a direct sum of irreducible representations of its $\operatorname{OSp}(1 \mid 2)_{\mathrm{ppal}}$.

Proposition $2[12,13]$. Any irreducible representation of $\mathrm{OSp}(1 \mid 2)$ is characterized by a quantum number $q=0, \frac{1}{2}, 1, \frac{3}{2}, \cdots$ and decomposes under its bosonic part $\mathrm{Sl}(2, R)$ into two multiplets $\left(q, q-\frac{1}{2}\right)$ for $q \neq 0$ - the case $q=0$ reducing to the trivial (one dimensional) representation.

We note that the dimension of irreducible $\operatorname{OSp}(1 \mid 2)$ representation $R(q)$ is $(4 q+1)=(2 q+1)+\left(2\left(q-\frac{1}{2}\right)+1\right)$.

Proposition $3[12,13]$. The product of two irreducible $\mathrm{OSp}(1 \mid 2)$ representations decomposes as follows:

$$
R\left(q_{1}\right) \times R\left(q_{2}\right)=\underset{q=\left|q_{1}-q_{2}\right|}{\bigoplus} R(q)
$$

$q$ taking integer and half-integer values.

As an aside comment, we remark that these properties confirm $\operatorname{OSp}(1 \mid 2)$ as the supersymmetric analogue of $\mathrm{Sl}(2)$.

We recall that $\mathrm{OSp}(1 \mid 2)_{\mathrm{ppal}}$ in $\mathscr{A}$ is generated from the fermionic root:

$$
F_{-\frac{1}{2}}=\sum_{i=1}^{r} E_{-\alpha_{i}}, r=\operatorname{rank} \mathscr{A}
$$

(we repeat that the $\mu_{i}$ coefficients in previous sections are irrelevant since they can be absorbed by rescaling of the $E_{\alpha_{i}}$ 's). Then the negative bosonic root appears as the sum of negative roots which are simple for the bosonic part of $\mathscr{A}$,

$$
X_{-}=\left\{F_{-\frac{1}{2}}, F_{-\frac{1}{2}}\right\}=\sum_{i=1}^{\tilde{r}} E_{-\beta_{i}}
$$


with $\tilde{r}=r$ for $\mathscr{A}=\operatorname{OSp}(2 n \pm 1 \mid 2 n), \operatorname{OSp}(2 n \mid 2 n)$ and $\operatorname{OSp}(2 n+2 \mid 2 n)$, and $\tilde{r}=r-1$ for $\mathscr{A}=\mathrm{Sl}(n+1 \mid n)$.

\subsection{Reduction of a Superalgebra into $\mathrm{OSp}(1 \mid 2)_{\mathrm{ppal}}$ Multiplets}

Let us consider separately each family of superalgebras admitting a fermionic S.R.S.

4.2.1. $\mathrm{Sl}(n+1 \mid n)$ case $(n \geqq 1)$. This is the simplest case. Indeed the $\mathrm{Sl}(n+1 \mid n)$ fundamental representation $(n+1) \oplus n$ reduces with respect to $\operatorname{OSp}(1 \mid 2)_{\text {ppal }}$ into the representation $R\left(\frac{n}{2}\right)=\left(D_{\frac{n}{2}}, \frac{D_{n-1}}{2}\right)$. The adjoint representation of $\mathrm{Sl}(n+1 \mid n)$ can be constructed from the direct product of the fundamental by its contragredient one (with respect to $\mathrm{Sl}(n+1) \oplus \mathrm{Sl}(n))$, then subtracting in the obtained result the trivial representation. Using the Prop. 3 above, one has

$$
R\left(\frac{n}{2}\right) \times R\left(\frac{n}{2}\right)=\sum_{q=0}^{q=n} R(q),
$$

where the sum goes over integer and half-integer $q$ 's. Therefore

$$
\mathrm{Sl}(n+1 \mid n) / \mathrm{OSp}(1 \mid 2)_{\mathrm{ppal}}=R(n) \oplus R\left(n-\frac{1}{2}\right) \oplus R(n-1) \oplus \ldots \oplus R\left(\frac{1}{2}\right) .
$$

Let us add that the Dynkin diagram of $\operatorname{Sl}(n+1 \mid n)$ relative to the S.R.S. is [9]

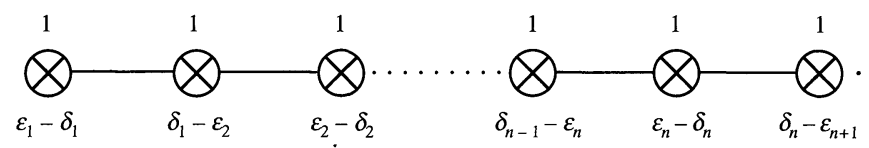

The $\operatorname{OSp}(1 \mid 2)_{\text {ppal }}$ is built from

$$
F_{+\frac{1}{2}}=E_{\varepsilon_{1}-\delta_{1}}+E_{\delta_{1}-\varepsilon_{2}}+\cdots+E_{\delta_{n}-\varepsilon_{n+1}},
$$

while the bosonic root is

$$
X_{+}=\left\{E_{+\frac{1}{2}}, E_{+\frac{1}{2}}\right\}=E_{\varepsilon_{1}-\varepsilon_{2}}+\cdots+E_{\varepsilon_{n}-\varepsilon_{n+1}}+E_{\delta_{1}-\delta_{2}}+\cdots+E_{\delta_{n-1}-\delta_{n}} .
$$

Moreover,

$$
h_{\max }=\frac{1}{2}(2 n)=n,
$$

that is the highest $q$ value in the above $R(q)$ decomposition.

4.2.2. OSp $(2 n-1 \mid 2 n)$ case $(n \geqq 1)$. The superalgebra $\operatorname{OSp}(2 n-1 \mid 2 n)$ contains as its bosonic part the algebra $\mathrm{O}(2 n-1) \oplus \mathrm{Sp}(2 n)$, and its fundamental representation reduces with respect to its bosonic part to $(2 n-1,1) \oplus(1,2 n)$. The $(2 n-1)$ fundamental of $\mathrm{O}(2 n-1)$ is irreducible with respect to the $\operatorname{OSp}(1 \mid 2)_{\text {ppal }}$ bosonic part, i.e. $\mathrm{Sl}(1,1)_{\text {ppal }}$ part, that is $(2 n-1) \equiv D_{n-1}$, as well as the $(2 n)$ representation of $\operatorname{Sp}(2 n):(2 n) \equiv D_{n-\frac{1}{2}}$.

The adjoint of $\mathrm{O}(2 n-1)$ can be obtained by taking the antisymmetric part in the product $(2 n-1) \times(2 n-1)$. In terms of $\mathrm{Sl}(2)$ representations, one can use the table of $\mathrm{O}(2 n-1)$ exponents [10] to get:

$$
D_{n-1} \times\left. D_{n-1}\right|_{A}=D_{2 n-3} \oplus D_{2 n-5} \oplus \cdots \oplus D_{1} .
$$


In the same way, the adjoint of $\mathrm{Sp}(2 n)$ can be constructed from the symmetric product of $(2 n) \times(2 n)$, and again the list of $\mathrm{Sp}(2 n)$ exponents provides the result:

$$
D_{n-\frac{1}{2}} \times D_{n-\frac{1}{2}}||_{S}=D_{2 n-1} \oplus D_{2 n-3} \oplus \cdots \oplus D_{1} .
$$

Fermionic generators in the adjoint of $\operatorname{OSp}(2 n-1 \mid 2 n)$ will be contained in the product $(2 n-1) \otimes(2 n)$, that is

$$
D_{n-1} \times D_{n-\frac{1}{2}}=D_{2 n-\frac{3}{2}} \oplus D_{2 n-\frac{5}{2}} \oplus \cdots \oplus D_{\frac{1}{2}} .
$$

Using Proposition 1 and Proposition 2, one can gather the Sl(2) representations of (4.9), (4.10) and (4.11) into $\mathrm{OSp}(1 \mid 2)$ ones in an unique way to obtain

$$
\begin{aligned}
& \mathrm{OSp}(2 n-1 \mid 2 n) / \mathrm{OSp}(1 \mid 2)_{\mathrm{ppal}}=R(2 n-1) \oplus R\left(2 n-\frac{5}{2}\right) \oplus R(2 n-3) \oplus \ldots \\
& \quad \ldots \oplus R(2(n-p)-1) \oplus R\left(2(n-p)-\frac{5}{2}\right) \oplus R(2(n-(p+1))-1) \oplus \ldots \\
& \quad \ldots \oplus R\left(\frac{3}{2}\right) \oplus R(1) .
\end{aligned}
$$

The Dynkin diagram associated to the fermionic S.R.S. is

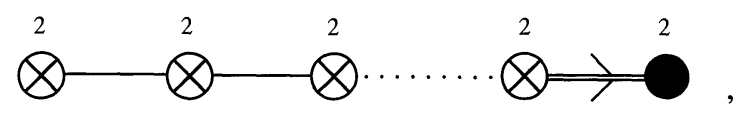

where there are $2(n-1)$ grey dots $(\otimes)$ and the black dot $(\Theta)$ corresponds to an $\mathrm{OSp}(1 \mid 2)$ fermionic root.

One notes that

$$
h_{\max }=\frac{1}{2}(2+\cdots+2)=2 n-1
$$

indeed corresponds to the highest $q$-value in the above $R(q)$ decomposition.

4.2.3. OSp $(2 n+1 \mid 2 n)$ case $(n \geqq 1)$. We can follow the same method as the one used just above, replacing $(2 n-1)$ by $(2 n+1)$. The result is

$$
\begin{aligned}
& \mathrm{OSp}(2 n+1 \mid 2 n) / \mathrm{OSp}(1 \mid 2)_{\mathrm{ppal}}=R\left(2 n-\frac{1}{2}\right) \oplus R(2 n-1) \oplus R\left(2 n-\frac{5}{2}\right) \oplus \cdots \\
& \quad \cdots \oplus R\left(2(n-p)-\frac{1}{2}\right) \oplus R(2(n-p)-1) \oplus R\left(2(n-(p+1))-\frac{1}{2}\right) \oplus \cdots \\
& \quad \cdots \oplus R\left(\frac{3}{2}\right) \oplus R(1) .
\end{aligned}
$$

The $\operatorname{OSp}(2 n+1 \mid 2 n)$ fermionic Dynkin diagram is

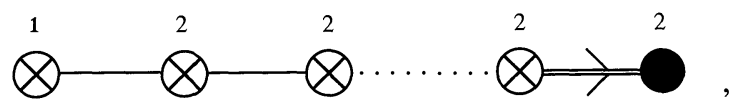

where there are $(2 n-1)$ grey dots. The value

$$
h_{\max }=\frac{1}{2}(2(2 n-1)+1)=2 n-\frac{1}{2}
$$

does correspond to the highest $R(q)$ representation.

4.2.4. $\mathrm{OSp}(2 n \mid 2 n)(n>1)$ case. We recall the isomorphism $\mathrm{OSp}(2 \mid 2) \cong \mathrm{Sl}(2 \mid 1)$. The reduction of the adjoint of $\mathrm{O}(2 n)$ and of $\mathrm{Sp}(2 n)$ with respect to $\mathrm{Sl}(2)_{\mathrm{ppal}}$ is given by the table of exponents [10] for these algebras

$$
\begin{gathered}
\mathrm{O}(2 n) \rightarrow D_{2 n-3} \oplus D_{2 n-5} \oplus \cdots \oplus D_{1} \oplus D_{n-1}, \\
\mathrm{Sp}(2 n) \rightarrow D_{2 n-1} \oplus D_{2 n-3} \oplus \cdots \oplus D_{1} .
\end{gathered}
$$


The $(2 n)$ fundamental representation of $\mathrm{Sp}(2 n)$ is irreducible under $\mathrm{Sl}(2)_{\mathrm{ppa}}$ : an indirect proof stands in the property of the $\mathrm{Sp}(2 n)$ adjoint representation to contain $D_{2 n-1}$ (see the exponent table [10] for example). Such a representation can only come from the product $D_{n-\frac{1}{2}} \times D_{n-\frac{1}{2}}$.

However, the $(2 n)$ of $\mathrm{O}(2 n)$ cannot be irreducible with respect to $\mathrm{Sl}(2)_{\mathrm{ppal}}$ : in such a case the $\operatorname{OSp}(2 n \mid 2 n)$ fermionic generators coming from $(2 n) \times(2 n)=D_{n-\frac{1}{2}} \times D_{n-\frac{1}{2}}$ would belong to $D_{j}$ representations with $j$ integer, and it would be impossible to reduce the $\operatorname{OSp}(2 n \mid 2 n)$ superalgebra into $\operatorname{OSp}(1 \mid 2)$ representations. But one needs the $\mathrm{O}(2 n)$ fundamental representation to contain $D_{n-1}$ in order to get $D_{1}$ by the product $D_{n-1} \times D_{n-\frac{1}{2}} \rightarrow D_{\frac{1}{2}}$ : this $D_{\frac{1}{2}}$ is necessary to reconstruct $\mathrm{OSp}(1 \mid 2)$ corresponding to $\left(D_{1}, D_{\frac{1}{2}}\right)$ in $\mathrm{OSp}(2 n \mid 2 n)$. Therefore for $\mathrm{O}(2 n)$ we have the $\mathrm{Sl}(2)_{\mathrm{ppal}}$ decomposition

$$
(2 n) \equiv D_{n-1} \oplus D_{0} .
$$

It follows that the fermions in $\operatorname{OSp}(2 n \mid 2 n)$ will belong to

$$
\left(D_{n-1} \oplus D_{0}\right) \times D_{n-\frac{1}{2}}=D_{2 n-\frac{3}{2}} \oplus D_{2 n-\frac{5}{2}} \oplus \cdots \oplus D_{\frac{1}{2}} \oplus D_{n-\frac{1}{2}},
$$

and we finally get

$$
\begin{aligned}
& \mathrm{OSp}(2 n \mid 2 n) / \mathrm{OSp}(1 \mid 2)_{\mathrm{ppal}}=R(2 n-1) \oplus R\left(2 n-\frac{5}{2}\right) \oplus R(2 n-3) \oplus \cdots \\
& \quad \cdots \oplus R(2(n-p)-1) \oplus R\left(2(n-p)-\frac{5}{2}\right) \oplus R(2(n-p-1)-1) \oplus \cdots \\
& \quad \cdots \oplus R\left(\frac{3}{2}\right) \oplus R(1) \oplus R\left(n-\frac{1}{2}\right) .
\end{aligned}
$$

The $\operatorname{OSp}(2 n \mid 2 n)$ fermionic Dynkin diagram is

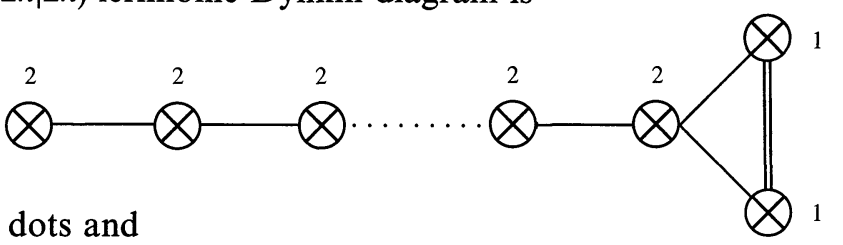

with $2 n$ grey dots and

$$
h_{\max }=\frac{1}{2}[2(2 n-2)+2]=2 n-1
$$

indeed corresponding to the highest $R(q)$ representation.

4.2.5. OSp $(2 n+2 \mid 2 n)$ case $(n \geqq 1)$. The reasoning is the same as for the $\operatorname{OSp}(2 n \mid 2 n)$ case, and the result is

$$
\begin{aligned}
& \mathrm{OSp}(2 n+2 \mid 2 n) / \mathrm{OSp}(1 \mid 2)_{\mathrm{ppal}}=R\left(2 n-\frac{1}{2}\right) \oplus R(2 n-1) \oplus R\left(2 n-\frac{5}{2}\right) \oplus \cdots \\
& \quad \cdots \oplus R\left(2(n-p)-\frac{1}{2}\right) \oplus R(2(n-p)-1) \oplus R(2(n-p-1)-1) \oplus \cdots \\
& \quad \cdots \oplus R\left(\frac{3}{2}\right) \oplus R(1) \oplus R(n) .
\end{aligned}
$$

We find again that

$$
h_{\max }=\frac{1}{2}[3+2(2 n-2)]=2 n-\frac{1}{2}
$$

in accordance with the value of $q$ in the above decomposition.

The OSp $(2 n+2 \mid 2 n)$ fermionic Dynkin diagram is

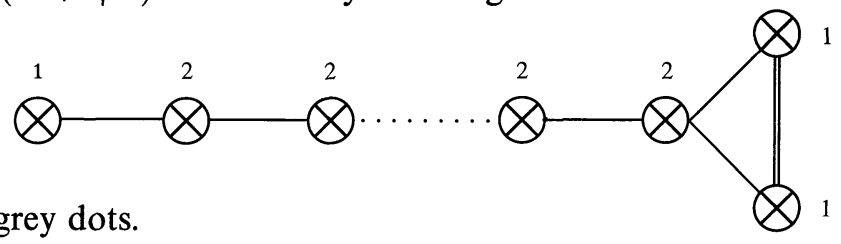

with $2 n+1$ grey dots. 
4.2.6. $D(2,1 ; \alpha)$ case $(\alpha \neq 0,-1)$. We get the same decomposition as for $\operatorname{OSp}(4 \mid 2)$, that is

$$
D(2,1 ; \alpha) / \mathrm{OSp}(1 \mid 2)_{\mathrm{ppal}}=2 R(1) \oplus R\left(\frac{3}{2}\right) .
$$

The Dynking diagram is now

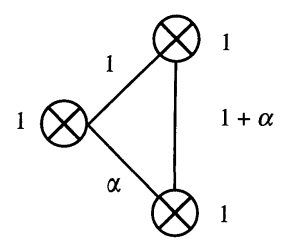

with

$$
h_{\max }=\frac{3}{2}
$$

as expected.

It would be of some interest to study the consequence of the $\alpha$ parameter on the corresponding super- $W$ algebra.

\section{The Spin Content of Super- $W$ Algebras}

We start by constructing the stress energy tensor of a super-Toda theory. Then, it is straightforward to deduce the conformal spin of a $W$ generator from the results of Sect. 4. It appears that the orthosympletic algebras are well-adapted to produce a generalization of the $N=1$ super-Virasoro algebra, while the $N=2$ superconformal algebra always shows up in the $\mathrm{Sl}(n+1 \mid n)$ theories. In this last case, we introduce the $\mathrm{Sl}(2 \mid 1)_{\mathrm{ppal}}$ superalgebra, containing $\operatorname{OSp}(1 \mid 2)_{\mathrm{ppal}}$, and better adapted to describe the group theoretical situation.

\subsection{Stress Energy Tensor for Super-WZW and Toda Theories}

In order to determine the superconformal spin of the $W$-generators, we have to construct the stress energy tensor of the theory. Let us first define ${ }^{1}$ :

$$
J^{\alpha}(X)=<J(X), T^{\alpha}>\text { with } X=(x ; \eta),
$$

$T^{\alpha}$ being any generator of the Lie superalgebra $\mathscr{A}$ under consideration. We also note

$$
\eta^{a b}=<T^{a}, T^{b}>=(-1)^{[a]} \eta^{b a},
$$

the scalar product already defined in $(2.2,2.3)$ with

$$
\eta^{a b} \eta_{b c}=\delta_{c}^{a}
$$

and the grading $[a]=0$ (respectively $[a]=1$ ) if $T^{a}$ is a commuting (respectively anticommuting) generator. Then

$$
J(X)=J_{a} T^{a}=(-1)^{[a]} J^{a} T_{a}, \text { where } J_{b}=J^{a} \eta_{a b}
$$

${ }^{1}$ From now on, we drop the Lorentz indices and write $(x, \eta)$ instead of $\left(x^{--}, \eta^{-}\right)$ 
and, for example

$$
\langle J(X), J(X)\rangle=J_{a} \eta^{a b} J_{b} .
$$

Do not forget at this point that $J(X)$ is a fermionic superfield, which implies $J^{a}(X)$ to have the grading $([a]+1)$.

Then we can set

$$
\left\{J^{a}(X), J^{b}(Y)\right\}_{\text {P.B. }}=i(-1)^{[a](1+[b])}\left(f_{c}^{a b} \delta(X-Y) J^{c}(Y)+\kappa \eta^{a b} D_{X} \delta(X-Y)\right),
$$

the $f_{c}^{a b}$ being the structure constants and $\kappa$ the parameter of the central extension of the Kac-Moody superalgebra $\mathscr{A}$. We point out that in the $T^{a}$ 's basis, the constants $f^{a b c}=f_{d}^{a b} \eta^{d c}$ are completely graded-antisymmetric, i.e.

$$
f^{a b c}=-(-)^{[a][b]} f^{b a c}=-(-)^{[b][c]} f^{a c b} .
$$

The super Dirac distribution is defined as

$$
\delta(X-Y)=\delta(x-y) \cdot(\eta-\theta) \quad \text { if } \quad X \equiv(x, \eta) \quad \text { and } \quad Y=(y, \theta)
$$

which implies in particular

$$
\begin{gathered}
\delta(X-Y)=-\delta(Y-X) \text { and } \\
\left\{J^{a}(X), J^{b}(Y)\right\}_{\text {P.B. }}=-(-1)^{(1+[a])(1+[b])}\left\{J^{b}(Y), J^{a}(X)\right\}_{\text {P.B. }} .
\end{gathered}
$$

After these preliminary measures, we can construct the super stress energy tensor. As in the non-supersymmetric case, it will be obtained by adding to the WZW part a correction term such that the current components constrained to be constant, have a vanishing superconformal spin.

The WZW stress energy tensor reads

with

$$
L_{\mathrm{wZw}}(X)=-\frac{1}{3 \kappa^{2}}\langle J(X), \hat{J}(X) J(X)\rangle-\frac{1}{2 \kappa}\langle J(X), D J(X)\rangle
$$

$$
\begin{aligned}
\left\{L_{\mathrm{WZW}}(X), J^{a}(Y)\right\}_{\text {P.B. }}= & i\left(\frac{1}{2} \partial_{x} \delta(X-Y) J^{a}(Y)\right. \\
& \left.-\delta(X-Y) \partial_{y} J^{a}(Y)-\frac{1}{2} D_{X} \delta(X-Y) D J^{a}(Y)\right),
\end{aligned}
$$

and finally we get

$$
L_{\text {Toda }}(X)=L_{\mathrm{wZW}}(X)-i D^{2}\langle H, J(X)\rangle,
$$

remembering $D^{2}=i \partial$ and $H$ given by (3.18).

Owing to the CR

$$
\left\{H(X), E_{h}(Y)\right\}_{\text {P.в. }}=i h \delta(X-Y) E_{h}(Y),
$$

where $h \in \frac{1}{2} Z \backslash\{0\}$ (cf. (3.17)), we get:

$$
\begin{aligned}
\left\{L_{\text {Toda }}(X), E_{h}(Y)\right\}_{\text {P.B. }}= & i\left(\left(\frac{1}{2}+h\right) \partial_{x} \delta(X-Y) E_{h}(Y)\right. \\
& \left.\left.-\delta(X-Y) \partial_{y} E_{h}(Y)-\frac{1}{2} D_{X} \delta(X-Y) E_{h}(Y)\right)\right) .
\end{aligned}
$$

One may remark that the Cartan components of the current are not primary fields in a Toda theory, while the other generators are. Since there is no Cartan generator showing up in $J^{g}$ given by (3.34), one concludes that the $W_{h_{i}}$ are primary super fields with superconformal spin $\left(h_{i}+\frac{1}{2}\right)$, that is $W_{h_{i}}$ contains one component with conformal spin $\left(h_{i}+\frac{1}{2}\right)$, and one component with spin $\left(h_{i}+1\right)$. 


\subsection{Orthosymplectic Superalgebras and the $N=1$ Case:}

We recall that to each $R(q)$ representation of $\operatorname{OSp}(1 \mid 2)$ appearing in the above decomposition is associated a $W$ generator with superconformal spin $\left(q+\frac{1}{2}\right)$, the two component fields being therefore of spin $\left(q+\frac{1}{2}\right),(q+1)$.

Let us first consider the spin content of the orthosymplectic algebras. We have:

$$
\begin{gathered}
\operatorname{OSp}(2 n-1 \mid 2 n):\left(\frac{3}{2}, 2\right)+\left(2, \frac{5}{2}\right)+\left(\frac{7}{2}, 3\right)+\ldots+\left(2 n-2,2 n-\frac{3}{2}\right)+\left(2 n-\frac{1}{2}, 2 n\right) \\
\operatorname{OSp}(2 n \mid 2 n):\left(\frac{3}{2}, 2\right)+\ldots+\left(2 n-2,2 n-\frac{3}{2}\right)+\left(2 n-\frac{1}{2}, 2 n\right)+\left(n, n+\frac{1}{2}\right) \\
\operatorname{OSp}(2 n+1 \mid 2 n):\left(\frac{3}{2}, 2\right)+\ldots+\left(2 n-2,2 n-\frac{3}{2}\right)+\left(2 n-\frac{1}{2}, 2 n\right)+\left(2 n, 2 n+\frac{1}{2}\right) \\
\operatorname{OSp}(2 n+2 \mid 2 n):\left(\frac{3}{2}, 2\right)+\ldots+\left(2 n-\frac{1}{2}, 2 n\right)+\left(2 n, 2 n+\frac{1}{2}\right)+\left(n+\frac{1}{2}, n+1\right) \\
D(2,1 ; \alpha) \alpha \neq 0,-1: 2\left(\frac{3}{2}, 2\right)+\left(2, \frac{5}{2}\right) .
\end{gathered}
$$

One remarks that the conformal spin content of $\operatorname{OSp}(2 n+1 \mid 2 n)$ is obtained by adding the spin multiplet $\left(2 n, 2 n+\frac{1}{2}\right)$ to the spin content of $\operatorname{OSp}(2 n-1 \mid 2 n)$. Similarly one goes from $\operatorname{OSp}(2 n+1 \mid 2 n)$ to $\operatorname{OSp}(2(n+1)-1 \mid 2(n+1))$ by adjunction of the conformal spin multiplet $\left(2 n+\frac{3}{2}, 2 n+2\right)$. These features of course reflect the embeddings $\operatorname{OSp}(2 n-1 \mid 2 n) \operatorname{COSp}(2 n+1 \mid 2 n) \operatorname{COSp}(2 n+1 \mid 2 n+2)$. In the same way, one has to add the spin multiplet $\left(n, n+\frac{1}{2}\right)$ to the $\operatorname{OSp}(2 n-1 \mid 2 n)$ spin multiplets to get the set of $\operatorname{OSp}(2 n \mid 2 n)$ spin multiplets, and the multiplet $\left(n+\frac{1}{2}, n+1\right)$ to those of $\operatorname{OSp}(2 n+1 \mid 2 n)$ to obtain the $\operatorname{OSp}(2 n+2 \mid 2 n)$ spin decomposition. In these cases, the associated embeddings are $\operatorname{OSp}(2 n-1 \mid 2 n) \subset \mathrm{OSp}(2 n \mid 2 n)$ and $\operatorname{OSp}(2 n+1 \mid 2 n) \operatorname{COSp}(2 n+2 \mid 2 n)$. As is well known the $\operatorname{OSp}(1 \mid 2)$ algebra has the correct spin content to build the $N=1$ Super Virasoro algebra $[5,14]$. Thus, all these orthosymplectic algebras which are permitted for Super-Toda theories furnish simple generalizations of the $N=1$ Super Virasoro algebra that is $N=1$ Super- $W$ algebras. We note in particular that there is, in any such a series, neither spin 1 nor spin $\frac{1}{2}$ element.

\section{3. $\mathrm{Sl}(n+1 \mid n)$ Superalgebras and the $N=2$ Case}

5.3.1. Introducing the $\mathrm{Sl}(2 \mid 1)_{\mathrm{ppal}}$ Superalgebra. For the $\mathrm{Sl}(n+1 \mid n)$ superalgebras, we have the following spin decomposition:

$$
\mathrm{Sl}(n+1 \mid n):\left(1, \frac{3}{2}\right)+\left(\frac{3}{2}, 2\right)+\ldots+\left(n, n+\frac{1}{2}\right)+\left(n+\frac{1}{2}, n+1\right),
$$

and one remarks that two spin multiplets, namely $\left(n+1, n+\frac{3}{2}\right)+\left(n+\frac{3}{2}, n+2\right)$ have to be joined to the spin content of $\operatorname{Sl}(n+1 \mid n)$ to obtain the $\operatorname{Sl}(n+2 \mid n+1)$ one. Indeed, considering the embedding $\operatorname{Sl}(n+1 \mid n) C \operatorname{Sl}(n+2 \mid n+1)$, one has to add the two OSp(1|2) representations $R\left(n+\frac{1}{2}\right)+R(n+1)$ to the adjoint representation of $\operatorname{Sl}(n+1 \mid n)$ to get that of $\operatorname{Sl}(n+2 \mid n+1)$. Actually, these two OSp $(1 \mid 2)$ representations constitute an irreducible one under a special $\mathrm{Sl}(2 \mid 1)$ subalgebra of $\mathrm{Sl}(n+2 \mid n+1)$ which we will call $\mathrm{Sl}(2 \mid 1)_{\text {ppal }}$. Let us define more precisely this algebra. set

Starting from the fermionic Dynkin diagram of $\mathrm{Sl}(n+1 \mid n)$ - see Sect. 4.2.1 - we

$$
\begin{gathered}
F_{+\alpha}=\sum_{i=1}^{n} E_{\delta_{i}-\varepsilon_{i}+1}, \quad F_{+\beta}=\sum_{i=1}^{n} E_{\varepsilon_{i}-\delta_{i}}, \\
F_{-\alpha}=\sum_{i=1}^{2 n} \sum_{j=1}^{n} K^{i(2 j)} E_{-\varepsilon_{j+1}+\delta_{j}}, \quad F_{-\beta}=\sum_{i=1}^{2 n} \sum_{j=1}^{n} K^{i(2 j-1)} E_{-\delta_{j}+\varepsilon_{j}}
\end{gathered}
$$


with $K^{i j}=\left(K^{-1}\right)_{i j}$ and:

$$
K=\left(\begin{array}{rrrrrrrr}
0 & 1 & & & & & \\
1 & 0 & -1 & & & & \\
& -1 & 0 & 1 & & & \\
& & 1 & 0 & & & \\
& & & & \ddots & 0 & 1 \\
& & & & & 1 & 0
\end{array}\right)
$$

It is now a simple exercise to verify that the algebra generated from $F_{ \pm \alpha}, F_{ \pm \beta}$ constitutes an $\mathrm{Sl}(2 \mid 1)$ algebra. Indeed

$$
\begin{gathered}
\left\{F_{ \pm \alpha}, F_{ \pm \alpha}\right\}=\left\{F_{ \pm \beta}, F_{ \pm \beta}\right\}=0, \\
\left\{F_{ \pm \alpha}, F_{ \pm \beta}\right\}=E_{ \pm}, \\
\left\{F_{+\alpha}, F_{-\alpha}\right\}=H_{++} H_{-}, \\
\text {with } H_{ \pm}=\frac{1}{2} \sum_{i=1}^{2 n} \sum_{j=1}^{n}\left(K^{i(2 j)} H_{2 j} \pm K^{i(2 j-1)} H_{2 j-1}\right), \\
\left\{F_{+\beta}, F_{-\beta}\right\}=H_{+}-H_{-}, \\
{\left[E_{ \pm}, F_{\mp \alpha}\right]=\mp F_{ \pm \beta},\left[E_{ \pm}, F_{\mp \beta}\right]=\mp F_{ \pm \alpha},} \\
{\left[E_{ \pm}, F_{ \pm \alpha}\right]=\left[E_{ \pm}, F_{ \pm \beta}\right]=0,}
\end{gathered}
$$

and

$$
\begin{aligned}
& {\left[H_{ \pm}, F_{\alpha}\right]= \pm \frac{1}{2} F_{\alpha},\left[H_{ \pm}, F_{\beta}\right]=\frac{1}{2} F_{\beta},} \\
& {\left[H_{ \pm}, F_{-\alpha}\right]=\mp \frac{1}{2} F_{-\alpha},\left[H_{ \pm}, F_{-\beta}\right]=-\frac{1}{2} F_{-\beta},} \\
& {\left[H_{+}, E_{ \pm}\right]= \pm E_{ \pm},\left[H_{-}, E_{ \pm}\right]=0 .}
\end{aligned}
$$

We call the subalgebra just defined $\mathrm{Sl}(2 \mid 1)_{\mathrm{ppa} 1}$ in $\mathrm{Sl}(n+1 \mid n)$. One immediately notes that: $\operatorname{Sl}(2 \mid 1)_{\text {ppal }}$ contains $\operatorname{OSp}(1 \mid 2)_{\text {ppal }}$ as a maximal subalgebra, with fermionic generators

$$
F_{ \pm \frac{1}{2}}=F_{ \pm \alpha}+F_{ \pm \beta}
$$

and bosonic generators $E_{ \pm}, H_{+}$.

More generally with respect to its $\mathrm{Sl}(2 \mid 1)_{\mathrm{ppal}}$, the adjoint representation of $\operatorname{Sl}(n+1 \mid n)$ reduces to

$$
\mathrm{S} 1(n+1 \mid n) / \mathrm{S} 1(2 \mid 1)_{\mathrm{ppal}}=\hat{R}(n) \oplus \hat{R}(n-1) \oplus \ldots \oplus \hat{R}(1),
$$

where we denote by $\hat{R}(m)$ - with $m$ integer - the 8 m-dimensional typical representation of $\operatorname{Sl}(2 \mid 1)$, the decomposition of which with respect to $\operatorname{OSp}(2 \mid 1)$ being:

$$
\hat{R}(m)=R(m) \oplus R\left(m-\frac{1}{2}\right) .
$$


The superalgebra $\mathrm{Sl}(2 \mid 1)$ corresponds to the $N=2$ Super-Virasoro case - or $N=2$ Ademollo et al. algebra - with spin $\left(1, \frac{3}{2}, \frac{3}{2}, 2\right)$.

As discussed in [15] the superalgebra $\mathrm{Sl}(3 \mid 2)$ does correspond to the super- $W_{3}$ algebra with spins $\left(1, \frac{3}{2}, \frac{3}{2}, 2,2, \frac{5}{2}, 3\right)$ and it is reasonable from the study of their spin content, to consider, following [16], the superalgebras $\mathrm{Sl}(n+1 \mid n)$ as corresponding to a direct generalization of the $N=2$ superconformal algebra. The just above defined $\mathrm{Sl}(2 \mid 1)_{\text {ppal }}$ algebra with its particular position in $\mathrm{Sl}(n+1 \mid n)$ appears, the $N=2$ case, as the analogous of $\operatorname{OSp}(1 \mid 2)_{\text {ppal }}$ for the $N=1$ case.

In this context, one can wonder whether the second Cartan generator $H_{-}$in $\mathrm{Sl}(2 \mid 1)$ could be associated to some grading, in the same way the generator $\mathrm{H}_{+}$, also in $\mathrm{OSp}(1 \mid 2)_{\text {ppal }}$, defines the grading (see (3.23)) related to the conformal spin. Actually, by a direct calculation, one can prove that:

$$
\begin{aligned}
{\left[H_{-}, E_{ \pm\left(\delta_{i}-\varepsilon_{i}+1\right)}\right] } & =\mp \frac{1}{2} E_{ \pm\left(\delta_{i}-\varepsilon_{i+1}\right)}, i \in\{1,2, \ldots, n\}, \\
{\left[H_{-}, E_{ \pm\left(\varepsilon_{i}-\delta_{i}\right)}\right] } & = \pm \frac{1}{2} E_{ \pm\left(\varepsilon_{i}-\delta_{i}\right)},
\end{aligned}
$$

while for any bosonic root $E_{\alpha}$ in $\operatorname{Sl}(n+1 \mid n)$ :

$$
\left[H_{-}, E_{\alpha}\right]=0 \text {. }
$$

It follows that for any fermionic generator $E_{\gamma}$ in $\operatorname{Sl}(n+1 \mid 1)$,

$$
\left[H_{-}, E_{\gamma}\right]=a(\gamma) E_{\gamma} \text { with } a(\gamma) \in\left\{-\frac{1}{2}, \frac{1}{2}\right\} \text {. }
$$

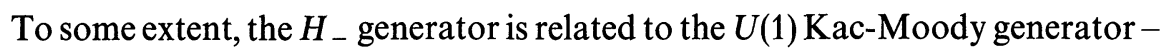
or spin 1 generator - appearing in the $N=2$ Super-Virasoro algebra.

Let us mention that our results for the spin content of $W$ algebras associated to super-Toda theories, agree with the tableaux given in [4] and guessed from spin values of low dimensional super- $W$ algebras.

Finally, let us remark that the existence of only one spin one in the $\operatorname{Sl}(n+1 \mid n)$ decomposition - and no spin one in the orthosymplectic cases - forbids the possibility to obtain super-Virasoro algebras with $N>2$ in this approach. For the super-Toda model based on $\operatorname{Sl}(n+1 \mid n)$, the $N=1$ superconformal tensor given in (5.14) is improved with the superspin 1 operator $W_{1}$ that contains the second supersymmetry generator together with the $U(1)$ Kac-Moody generator. The construction of the $W_{1}$ operator goes as follows:

5.3.2. The Super- $W_{1}$ Generator. Since the $W_{1}$ operator is the lowest spin generator, it is easy to obtain it from the general method of Sect.3.3.

We first execute on $J$ a gauge transformation (3.12) based on negative simple roots

$$
g_{-\frac{1}{2}}=\exp \left(\sum_{\alpha \in \Delta_{+}} \Omega^{\alpha} E_{-\alpha}\right)
$$

so that the coefficients of all the Cartan generators in $\operatorname{Ad}^{*}\left(g_{-\frac{1}{2}}\right)(J)$ are set to zero. Then, a second gauge transformation

$$
g_{-1}=\exp \left(\sum_{\alpha \in \Pi^{(+1)}} \Omega^{\alpha} E_{-\alpha}\right)
$$

is used to cancel all the coefficients of the (negative) simple roots generators, except the one associated to a lowest weight of $\operatorname{OSp}(1 \mid 2)_{\text {ppal }}$, and which is exactly $W_{1}$. In order to give a compact form for $W_{1}$, we first define a linear transformation $\tau$ which 
acts on a general element of $\operatorname{Sl}(n+1 \mid n)$,

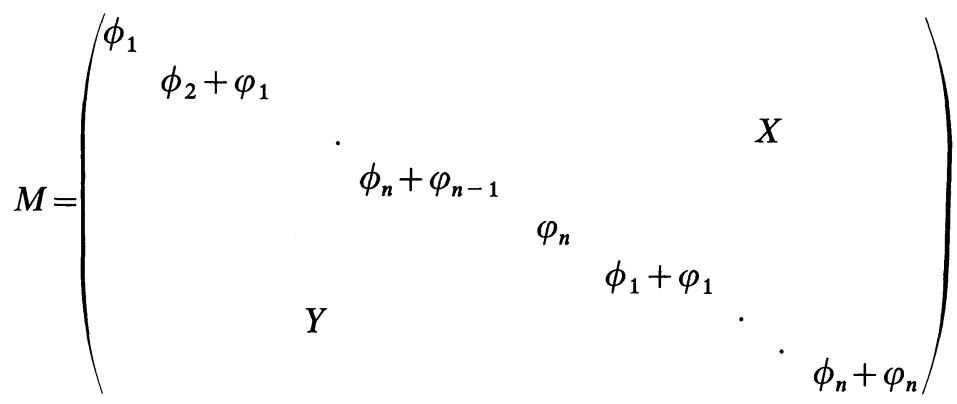

as

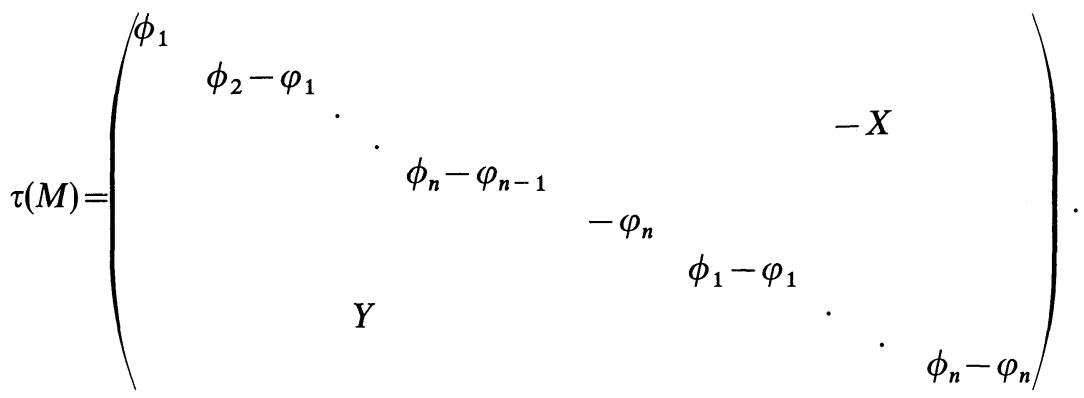

This transformation is not an automorphism of the superalgebra, but has the following properties ${ }^{2}$ :

$$
\begin{aligned}
& \tau^{2}=\mathrm{Id}, \\
& \operatorname{Str}(\tau(M) \tau(N))=-\operatorname{Str}(M N), \\
& {[\tau(M), \tau(N)\}-\tau([\tau(M), N\})-\tau([M, \tau(N)\})+[M, N\}=0 .}
\end{aligned}
$$

$\tau$ makes explicit the role played by $\mathrm{S} 1(2 \mid 1)_{\mathrm{ppal}}$ in the $N=2$ case: actually, the smallest Lie algebra containing $\tau\left(\mathrm{OSp}(1 \mid 2)_{\text {ppal }}\right)$ is just $\mathrm{Sl}(2 \mid 1)_{\text {ppal }}$, the action of $\tau$ on the generator of this superalgebra being given by (the notations are the same as in Sect. 5.3.1)

$$
\begin{array}{cll}
\tau\left(F_{\alpha}\right)=-F_{\alpha}, & \tau\left(F_{-\alpha}\right)=F_{-\alpha}, & \tau\left(F_{\beta}\right)=F_{\beta}, \quad \tau\left(F_{-\beta}\right)=-F_{-\beta}, \\
\tau\left(H_{+}\right)=H_{-}, & \tau\left(H_{-}\right)=H_{+} .
\end{array}
$$

In fact, the transformation $i \tau$ is a complex structure [17] on the compact supergroup generated by $\operatorname{Sl}(n+1 \mid n)$, and it is not surprising that it appears in the expression of $W_{1}$,

\section{Examples}

$$
W_{1}=\frac{1}{4} \operatorname{Str}(J \tau(J))-\frac{1}{2} \operatorname{Str}\left(H_{-} D J\right)
$$

Let us apply the general properties discussed above on the two simplest and basic examples.

\footnotetext{
${ }^{2}$ We recall that for the $\mathrm{Sl}(n+1 \mid n)$ series, the super-trace operator is proportional to $<,>$
} 


\subsection{Study of $\operatorname{OSp}(1 \mid 2)$}

$\operatorname{OSp}(1 \mid 2)$ is generated by two fermions $F_{ \pm}$and a bosonic $\operatorname{Sl}(2)\left(H, E_{+}, E_{-}\right)$. Its commutation relation reads ${ }^{3}$

$$
\begin{array}{cc}
{\left[H, E_{ \pm}\right]= \pm E_{ \pm},} & {\left[H, F_{ \pm}\right]= \pm \frac{1}{2} F_{ \pm},} \\
{\left[E_{+}, F_{-}\right]=F_{+},} & {\left[E_{-}, F_{+}\right]=F_{-},} \\
{\left[E_{+}, E_{-}\right]=2 H,} & \left\{F_{+}, F_{-}\right\}=-\frac{1}{2} H, \\
\left\{F_{+}, F_{+}\right\}=\frac{1}{2} E_{+}, & \left\{F_{-}, F_{-}\right\}=-\frac{1}{2} E_{-} .
\end{array}
$$

Using the 3-dimensional representation

$$
\begin{gathered}
H=\left(\begin{array}{rrr}
\frac{1}{2} & 0 & \\
0 & -\frac{1}{2} & 0 \\
0 & 0 & 0
\end{array}\right), \quad E_{+}=\left(\begin{array}{lll}
0 & 1 & 0 \\
0 & 0 & 0 \\
0 & 0 & 0
\end{array}\right), \quad E_{-}=\left(\begin{array}{lll}
0 & 0 & 0 \\
1 & 0 & 0 \\
0 & 0 & 0
\end{array}\right), \\
F_{+}=\left(\begin{array}{lll}
0 & 0 & \frac{1}{2} \\
0 & 0 & 0 \\
0 & \frac{1}{2} & 0
\end{array}\right), \quad F_{-}=\left(\begin{array}{rrr}
0 & 0 & 0 \\
0 & 0 & \frac{1}{2} \\
-\frac{1}{2} & 0 & 0
\end{array}\right) .
\end{gathered}
$$

One gets for the constraints current $J$,

$$
J=\left(\begin{array}{ccc}
\Phi & 0 & \mu \\
Y & -\Phi & -U \\
U & \mu & 0
\end{array}\right)
$$

$\Phi, Y$ being fermionic and $U$ a bosonic superfield and a constraint $\left\langle J, F_{-}\right\rangle$ $=2 \mu=C^{\text {st }}$. An element of the residual gauge group then takes the form

$$
\mathrm{G}=\exp \left(A E_{-}+2 \Omega F_{-}\right)=\left(\begin{array}{ccc}
1 & 0 & 0 \\
A & 1 & \Omega \\
-\Omega & 0 & 1
\end{array}\right)
$$

$A$ (respectively $\Omega$ ) being bosonic (respectively fermionic) $G$ acts on $J$ as:

$$
\begin{aligned}
\operatorname{Ad}(A, \Omega)(J)=\hat{G} J G^{-1}+(D G) G^{-1} & \\
= & \left(\begin{array}{ccc}
\Phi+\mu \Omega & 0 & \mu \\
Y+2(A \Phi-\Omega U+\mu \Omega A)+D A+\Omega D \Omega & -(\Phi+\mu \Omega) & U+\Omega \Phi-\mu A-D \Omega \\
U+\Omega \Phi-\mu A-D \Omega & \mu & 0
\end{array}\right) .
\end{aligned}
$$

With the special values:

\footnotetext{
${ }^{3}$ Be careful of the change of normalisation $J_{ \pm}= \pm 2 F_{ \pm}, X_{ \pm}= \pm E_{ \pm}$
} 
we get the invariant supercurrent

$$
\mathrm{J}^{g}=\operatorname{Ad}\left(A_{0}, \Omega_{0}\right)(J)=\left(\begin{array}{ccc}
0 & 0 & \mu \\
W_{3 / 2} & 0 & 0 \\
0 & \mu & 0
\end{array}\right)
$$

with $W_{3 / 2}=Y+\frac{1}{\mu^{2}}\left(\Phi D \Phi+\mu D U+2 \mu \Phi U+D^{2} \Phi\right)$.

Note that the invariance of $J^{g}$ under the residual gauge transformations can be explicitly checked thanks to

$$
\begin{gathered}
\operatorname{Ad}\left(A_{1}, \Omega_{1}\right) \operatorname{Ad}\left(A_{2}, \Omega_{2}\right)=\operatorname{Ad}\left(A_{1}+A_{2}-\Omega_{1} \Omega_{2}, \Omega_{1}+\Omega_{2}\right) \\
\operatorname{Ad}(A, \Omega) A_{0}=A_{0}-A-\Omega \Omega_{0} \\
\operatorname{Ad}(A, \Omega) \Omega_{0}=\Omega_{0}-\Omega
\end{gathered}
$$

Finally, $W_{3 / 2}$ can be identified with the super-Virasoro generator via the formula:

$$
W_{3 / 2}=\frac{-1}{\mu^{2}} \operatorname{Str}\left(\frac{1}{3} J \hat{J} J+\frac{1}{2} J D J-H D^{2} J\right)
$$

with the supertrace operator (Str) proportional to the scalar product $\langle$, $\rangle$.

\subsection{Case of $\mathrm{Sl}(2 \mid 1)$}

The fundamental representation of $\mathrm{Sl}(2 \mid 1)$ is 3-dimensional:

$$
\begin{array}{ccc}
H_{1}=\left(\begin{array}{ccc}
1 / 2 & 0 & 0 \\
0 & -1 / 2 & 0 \\
0 & 0 & 0
\end{array}\right), & H_{2}=\left(\begin{array}{ccc}
1 / 2 & 0 & 0 \\
0 & 1 / 2 & 0 \\
0 & 0 & 1
\end{array}\right), \\
E_{+}=\left(\begin{array}{ccc}
0 & 1 & 0 \\
0 & 0 & 0 \\
0 & 0 & 0
\end{array}\right), & E_{-}=\left(\begin{array}{ccc}
0 & 0 & 0 \\
1 & 0 & 0 \\
0 & 0 & 0
\end{array}\right), \\
F_{++}=\left(\begin{array}{lll}
0 & 0 & 0 \\
0 & 0 & 0 \\
0 & 1 & 0
\end{array}\right), & F_{+-}=\left(\begin{array}{ccc}
0 & 0 & 1 \\
0 & 0 & 0 \\
0 & 0 & 0
\end{array}\right), \\
F_{-+}=\left(\begin{array}{lll}
0 & 0 & 0 \\
0 & 0 & 0 \\
1 & 0 & 0
\end{array}\right), & F_{--}=\left(\begin{array}{lll}
0 & 0 & 1 \\
0 & 0 & 0
\end{array}\right) .
\end{array}
$$

The constraints $\left\langle J, F_{--}\right\rangle=-\mu_{1},\left\langle J, F_{-_{+}}\right\rangle=\mu_{2}$, and $\left\langle J, E_{-}\right\rangle=0$ lead to

$$
J=\left(\begin{array}{ccc}
\Phi_{1} & 0 & \mu_{2} \\
Y & \Phi_{2} & U_{2} \\
U_{1} & \mu_{1} & \Phi_{1}+\Phi_{2}
\end{array}\right) \text { with } \begin{aligned}
& \Phi_{1}, \Phi_{2}, Y \text { fermionic } \\
& U_{1}, U_{2} \text { bosonic }
\end{aligned}
$$


The residual gauge elements

act on $J$ as

$$
G=\exp \left(A E_{-}+\Omega F_{-_{+}}+\Sigma F_{-_{-}}\right)=\left(\begin{array}{ccc}
1 & 0 & 0 \\
A+\frac{1}{2} \Sigma \Omega & 1 & \Sigma \\
\Omega & 0 & 1
\end{array}\right)
$$

$$
\begin{aligned}
& \operatorname{Ad}(A, \Omega, \Sigma)(J) \\
& =\left(\begin{array}{c}
\Phi_{1}-\mu_{2} \Omega \\
Y-\Sigma U_{1}-\Omega U_{2}+\left(A-\frac{1}{2} \Sigma \Omega\right)\left(\Phi_{1}-\mu_{2} \Omega\right)-\left(A+\frac{1}{2} \Sigma \Omega\right)\left(\Phi_{2}-\mu_{1} \Sigma\right) \\
U_{1}+\Omega\left(\Phi_{2}-\mu_{1} \Sigma\right)-\mu_{1}\left(A+\frac{1}{2} \Sigma \Omega\right) \\
\mu_{2} \\
\Phi_{2}-\mu_{1} \Sigma \\
\mu_{1}-\Sigma\left(\Phi_{1}-\mu_{2} \Omega\right)+\mu_{2}\left(A-\frac{1}{2} \Sigma \Omega\right) \\
\Phi_{1}+\Phi_{2}-\mu_{1} \Omega-\mu_{2} \Sigma
\end{array}\right) \\
& +\left(\begin{array}{ccc}
0 & 0 & 0 \\
D A-\frac{1}{2}((D \Sigma) \Omega+\Sigma D \Omega) & 0 & D \Sigma \\
D & 0 & 0
\end{array}\right)
\end{aligned}
$$

with $A$ (respectively $\Omega, \Sigma$ ) bosonic (respectively fermionic).

Again, for the special transformation $A_{0}=\frac{1}{2 \mu_{1} \mu_{2}}\left(\mu_{2} U_{1}-\mu_{1} U_{2}+D \Phi_{1}-D \Phi_{2}\right)$, $\Omega_{0}=\frac{1}{\mu_{2}} \Phi_{1}$ amd $\Sigma_{0}=\frac{1}{\mu_{1}} \Phi_{2}$ we obtain the invariant current

$$
J_{0}=\operatorname{Ad}\left(A_{0}, \Omega_{0}, \Sigma_{0}\right) J=\left(\begin{array}{ccc}
0 & 0 & \mu_{2} \\
W_{3 / 2} & 0 & \frac{1}{\mu_{1}} W_{1} \\
\frac{1}{\mu_{2}} W_{1} & \mu_{1} & 0
\end{array}\right)
$$

with

$$
\left\{\begin{aligned}
W_{3 / 2}= & \frac{1}{2 \mu_{1} \mu_{2}}\left(2 \mu_{1} \mu_{2} Y-2 \mu_{2} \Phi_{2} U_{1}-2 \mu_{1} \Phi_{1} U_{2}\right. \\
& \left.-\Phi_{1} D \Phi_{2}-\Phi_{2} D \Phi_{1}+\mu_{2} D U_{1}-\mu_{1} D U_{2}+D^{2}\left(\Phi_{1}-\Phi_{2}\right)\right) \\
W_{1}= & \frac{1}{2}\left(\mu_{2} U_{1}+\mu_{1} U_{2}+\Phi_{1} \Phi_{2}+D \Phi_{1}+D \Phi_{2}\right)
\end{aligned}\right.
$$

that is also

$$
\left\{\begin{array}{l}
W_{3 / 2}=\frac{-1}{\mu_{1} \mu_{2}} \operatorname{Str}\left(\frac{1}{3} J \hat{J} J+\frac{1}{2} J D J-H_{1} D^{2} J\right) \\
W_{1}=\operatorname{Str}\left(\frac{1}{4} J \tau(J)-\frac{1}{2} H_{2} D J\right)
\end{array}\right.
$$


where $\tau$ is defined as (see Sect. 5.3.2):

$$
\tau\left(\begin{array}{ccc}
\Phi_{1} & 0 & \mu_{2} \\
Y & \Phi_{2} & U_{2} \\
U_{1} & \mu_{1} & \Phi_{1}+\Phi_{2}
\end{array}\right)=\left(\begin{array}{ccc}
\Phi_{1} & 0 & -\mu_{2} \\
Y & -\Phi_{2} & -U_{2} \\
U_{1} & \mu_{1} & \Phi_{1}-\Phi_{2}
\end{array}\right) .
$$

\section{Conclusion}

Using an $N=1$ superfield formalism we have shown that Super-Toda theories are constrained super-WZW models associated with superalgebras admitting a completely fermionic simple root system. Carrying a constrained super-WZW model leads to reduce the corresponding superalgebra with respect to its $\operatorname{OSp}(1 \mid 2)$ principal embedding. To each $\operatorname{OSp}(1 \mid 2)_{\text {ppal }}$ representation showing up in this decomposition is attached a super- $W$ generator belonging to the symmetry algebra of this super Toda theory, or super- $W$ algebra. The $\mathrm{Sl}(n+1 \mid n)$ series provides a family of $N=2$ super- $W$ algebras, whereas the other series lead to $N=1$ super- $W$ algebras. We remark that if the classification is governed by the $\operatorname{OSp}(1 \mid 2)_{\text {ppal }}$ algebra in the orthosymplectic series, it better stands on a bigger subalgebra, that we have denoted $\operatorname{Sl}(2 \mid 1)_{\text {ppal }}$ and which contains $\operatorname{OSp}(1 \mid 2)_{\text {ppal }}$, in the $\mathrm{Sl}(n+1 \mid n)$ case. As examples, we explicitly construct the $N=1$ super-Virasoro algebra from the $\operatorname{OSp}(1 \mid 2)$ theory and the $N=2$ Ademollo et al. algebra from the $\mathrm{Sl}(2 \mid 1)$ theory.

Among the developments we can think of, let us first mention the $N=2$ supersymmetric case, in which the algebra $\mathrm{Sl}(2 \mid 1)_{\text {ppal }}$ has a role to play. New types of super- $W$ algebras will also be provided by constructing non-Abelian [18] - or generalized [19] - super-Toda theories, then limiting the constraints to a subset of the original ones. Technically, the different $\operatorname{OSp}(1 \mid 2)$ embeddings in the superalgebra of the theory will have to be studied, each of them giving rise to a different super- $W$ algebra: these $\operatorname{OSp}(1 \mid 2)$ embeddings replace the $\mathrm{Sl}(2)$ ones for a non-Abelian non-supersymmetric Toda theory [18, 19].

Finally, from a simple group theoretical point of view, our study deeply shows that the $\operatorname{OSp}(1 / 2)_{\text {ppal }}$ embedding in a simple superalgebra plays a role analogous to the one of the $\mathrm{Si}(2)_{\mathrm{ppal}}$ in an algebra. Our results suggest that a general relation exists between the OSp $(1 \mid 2)_{\text {ppal }}$ subalgebra and the Casimir degrees of a superalgebra. Such a property would generalize the result of [20] for algebras.

\section{References}

1. Balog, J., Feher, L., Forgacs, P., O'Raifeartaigh, L., Wipf, A.: Phys. Lett. B 227, 214 (1989); Phys. Lett B 244, 435 (1990); Ann. Phys. 203, 76 (1990)

2. Leites, D.A., Saveliev, M.V., Serganova, V.V.: In: Group theoretical methods in physics. Amsterdam: North-Holland 1985; preprint IHEP 85-81, Serpukhov 1985

3. Nohara, H., Mohri, K.: Nucl. Phys. B 349, 253 (1991)

4. Komata, S., Mohri, K., Nohara, H.: Nucl. Phys. B 359, 168 (1991)

5. Evans, J., Hollowood, T.: Nucl. Phys. B 352, 723 (1991)

6. Inami, T., Izawa, K.I.: Phys. Lett. B 255, 521 (1991)

7. Kac, V.: Adv. Math. 26, 8 (1977)

8. Van der Leur, J.W.: Contragredient Lie superalgebras of finite growth. Utrecht thesis (1985)

9. Frappat, L., Sciarrino, A., Sorba, P.: Commun. Math. Phys. 121, 457 (1989) 
10. Drinfeld, V.G., Sokolov, V.V.: J. Sov. Math. 30, 1975 (1985)

11. Leznov, A.N., Savelie, M.V.: Sov. J. Theor. Math. Phys. 61, 150 (1984)

12. Scheunert, M., Nahm, W., Rittenberg, V.: J. Math. Phys. 18, 155 (1977) and references therein

13. Berezin, F.A., Tolstoy, V.N.: Commun. Math. Phys. 78, 409 (1981)

14. Arvis, J.F.: Nucl. Phys. B 212, 151 (1983); B 218, 309 (1983)

Babelon, O.: Nucl. Phys. B 258, 680 (1985)

Babelon, O., Langouche, F.: Nucl. Phys. B 290, 603 (1987)

Leznov, A.N., Savelier, M.V., Leites, D.A.: Phys. Lett. B 96, 97 (1980)

Chaichian, M., Kulish, P.P.: Phys. Lett. B 78, 413 (1978)

Liao, H.C., Mansfield, P.: Nucl. Phys. B 344, 696 (1990)

15. Romans, L.J.: Preprint USC-91/HEP 06

Lu, H., Pope, C.N., Romans, L.J., Shen, X., Wang, X-J.: Phys. Lett. B 264, 91 (1991)

16. Nemeschansky, D., Yankielowicz, S.: Preprint TAUP 1860-91, USC-91/005A

17. Spindel, Ph., Sevrin, A., Troost, W., Van Proeyen, A.: Phys. Lett. B 206, 71 (1988)

18. Leznov, A.N., Saveliev, M.V.: Commun. Math. Phys. 89, 59 (1983)

Saveliev, M.V.: Mod. Phys. Let. 5A, 2223 (1990) and references therein

19. O'Raifeartaigh, L., Ruelle, P., Tsutsui, I., Wipf, A.: Preprint ETH-TH/91-4, DIAS-STP-91-02

20. Kostant, B.: Adv. Math. 34, 195 (1959)

Communicated by J. Fröhlich 